Revue des patrimoines

\title{
Le quartier et la tour de l'Europe à Mulhouse (1959-2015). Perspectives européennes d'un patrimoine transfrontalier
}

The European quarter and the Tour de l'Europe at Mulhouse, European

perspectives on a cross-border heritage (1959-2015)

\section{Amandine Diener}

\section{(2) OpenEdition}

Journals

Édition électronique

URL : http://journals.openedition.org/insitu/20178

DOI : $10.4000 /$ insitu. 20178

ISSN : $1630-7305$

Éditeur

Ministère de la culture

Référence électronique

Amandine Diener, « Le quartier et la tour de l'Europe à Mulhouse (1959-2015). Perspectives

européennes d'un patrimoine transfrontalier », In Situ [En ligne], 38 | 2019, mis en ligne le 15 février

2019, consulté le 19 avril 2019. URL : http://journals.openedition.org/insitu/20178 ; DOI : 10.4000/ insitu. 20178

Ce document a été généré automatiquement le 19 avril 2019

\section{c) (†)}

In Situ Revues des patrimoines est mis à disposition selon les termes de la licence Creative Commons Attribution - Pas d'Utilisation Commerciale - Pas de Modification 4.0 International. 


\title{
Le quartier et la tour de l'Europe à Mulhouse (1959-2015). Perspectives européennes d'un patrimoine transfrontalier
}

\author{
The European quarter and the Tour de l'Europe at Mulhouse, European \\ perspectives on a cross-border heritage (1959-2015)
}

Amandine Diener

$1 \mathrm{Au} \mathrm{XIX}{ }^{\mathrm{e}}$ siècle, la ville de Mulhouse s'est façonné un destin industriel audacieux, notamment grâce aux initiatives de la Société industrielle de Mulhouse (SIM), fondée en 1824, et à l'impulsion de Nicolas Koechlin (1751-1852), indienneur et député du Haut-Rhin ${ }^{1}$. En quelques décennies, la ville et la région industrielle qui l'entoure se dotent en effet d'une puissante infrastructure économique et se soudent au territoire national. Le patronat, véritable moteur de réalisations industrielles et sociales, suscitent l'admiration au-delà des frontières. Symbole de l'explosion industrielle, surnommée «le Manchester français ", Mulhouse fait figure de ville d'avant-garde.

2 La phase brillante de l'histoire de la ville ne résiste pas à la crise économique de 1930 et la Seconde Guerre mondiale brise l'élan créateur des Mulhousiens. La ville vit plus durement qu'ailleurs les profondes et difficiles mutations des économies européennes. Les friches disséminées au centre et à la périphérie offrent l'occasion de lancer de nouveaux projets d'urbanisme. Émile Muller (1915-1988), maire entre $1959^{2}$ et 1981, fait le pari de changer la ville par l'urbanisme et l'architecture; le projet de reconversion du quartier de la Dentsche en un quartier Europe, signé par l'architecte mulhousien François Spoerry (1912-1999) associé à Bernard Michau (1928-), en est l'un des premiers éléments moteurs.

3 Cet article retrace la genèse de ce projet, dans ses dimensions tant architecturales et urbaines que politiques. Il revient sur l'entreprise menée par ses concepteurs, notamment la collaboration entre le maire et l'urbaniste, pour revitaliser une ville désindustrialisée 
et lui donner un nouveau rayonnement européen par le biais d'un projet urbain dont la tour serait un élément phare de la composition. Il interroge, enfin, les limites d'un projet qui, après avoir subi de vives critiques, connait peu à peu un lent processus de patrimonialisation. La littérature, assez peu fournie sur l'histoire de la ville de Mulhouse depuis la Seconde Guerre mondiale ${ }^{3}$, l'est tout aussi peu à propos du projet d'aménagement urbain de la Dentsche. Hormis quelques brèves notes descriptives ${ }^{4}$, la première étude qui lui ait été consacrée est un récent mémoire universitaire ${ }^{5}$. En complément, les fonds d'archives de la société anonyme (S.A.) la Dentsche ${ }^{6}$ et des architectes François Spoerry ${ }^{7}$ et Bernard Michau ${ }^{8}$ ont été exploités - tous trois conservés aux archives de la Ville de Mulhouse - ainsi que quelques articles ${ }^{9}$ et numéros de revues ${ }^{10}$ consacrés à l'histoire de la ville et au projet du quartier Europe.

\section{Changer la ville par l'urbanisme. Émile Muller et François Spoerry : le maire et l'urbaniste}

Alors que s'achèvent les chantiers de reconstruction, des formes neuves d'urbanisation émergent ${ }^{11}$ sous l'égide du ministère de la Reconstruction et de l'Urbanisme (MRU), créé en 1944. Sur le plan politique, le Mulhouse des Trente Glorieuses est dominé par la figure d'Émile Muller qui cumule les fonctions de maire (1959-1981), député du Haut-Rhin (1958-1981) et conseiller général de Mulhouse-Nord (1958-1982). Son action en faveur du renforcement du centre-ville caractérise son premier mandat. À côté des deux centres existants de la ville - d'un côté la place de la Réunion, où se trouve l'ancien hôtel de ville $\mathrm{du} \mathrm{Xv}^{\mathrm{e}}$ siècle, qui symbolise l'époque de la République indépendante de Mulhouse, sous l'Ancien Régime, de l'autre la place de la Bourse dont le plan, dessiné par les architectes Geoffroy Stotz (1799-1831) et Félix Fries (1801-1859), témoigne de la grandeur industrielle de la ville au XIX ${ }^{e}$ siècle ${ }^{12}$ - le site de la Dentsche représente la possibilité d'une centralité nouvelle incarnant le Mulhouse moderne. Le maire fait ainsi le choix du dédoublement du centre ; la colonisation de la friche industrielle de la Dentsche ${ }^{13}$ laisse place à un nouveau cœur de béton situé au carrefour de la vieille ville. 
Figure 1

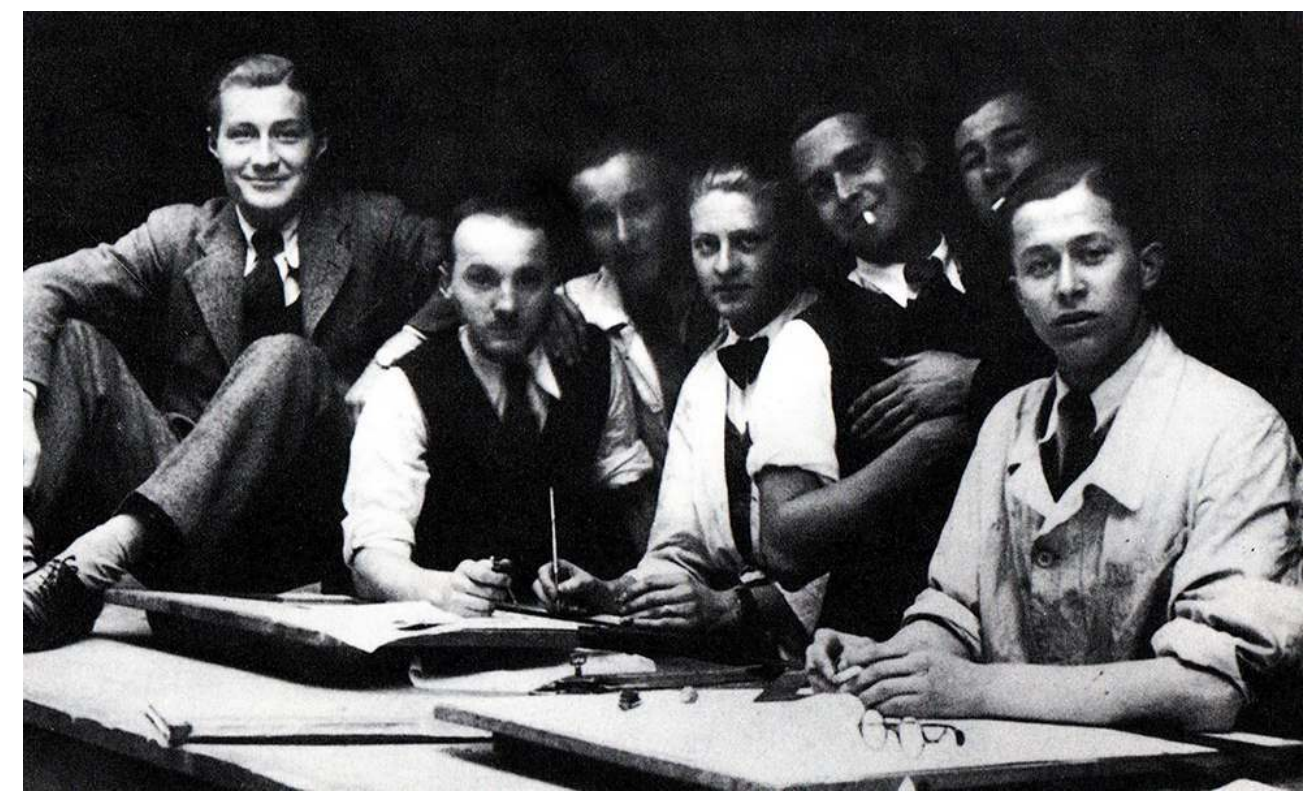

François Spoerry au côté de ses camarades à l'école régionale d'Architecture de Strasbourg en 1930. De gauche à droite au premier plan : François Spoerry (à gauche assis sur la table), Jean Hatt, Robert Mayer et René Schmitt. Phot. anonyme. Collection particulière (SPOERRY, François. L'Architecture douce, de Port-Grimaud à Port-Liberté. France, Ed. Laffont, 1989, p. 23).

Pour mener à bien sa politique, Muller s'entoure d'une équipe municipale fidèle dans laquelle figure Spoerry. Ce dernier, formé à l'architecture entre Strasbourg (fig. 1), Paris et Marseille ${ }^{14}$, développe une partie de son activité professionnelle à Mulhouse, sa ville d'origine, tout en assurant, de 1959 à 1981, les fonctions de conseiller municipal auprès d'Émile Muller ${ }^{15}$. L'affinité entre les deux hommes et l'engagement de Spoerry sur le plan professionnel et politique valent certainement à ce dernier un rôle de premier plan dans le projet d'aménagement du quartier de la Dentsche. Pour cet important réaménagement urbain, la Ville se dispense d'organiser un concours d'architecture et d'urbanisme et décide de « charger un architecte de renom de l'établissement des projets définitifs ${ }^{16}$ ». En 1957, François Spoerry est désigné architecte-urbaniste conseil ainsi que Jean-Henri Calsat (1905-1991), celui-ci s'étant déjà vu confier par le MRU, en 1950, l'élaboration du plan du groupement d'urbanisme de Strasbourg ${ }^{17}$. Selon les termes de leur contrat, ils sont chargés « des études et de l'établissement des plans d'aménagement et plan-masse, ainsi que des programmes et règlements en dépendant » et de mettre au point « les plans d'aménagement et les plans-masses du quartier ${ }^{18} »$. Certains dispositifs spatiaux sont exigés par le programme tel qu'une "place oblongue de forme trapézoïdale [...] [dont la base sera] occupée par un édifice à caractère monumental ${ }^{19}$ ». 


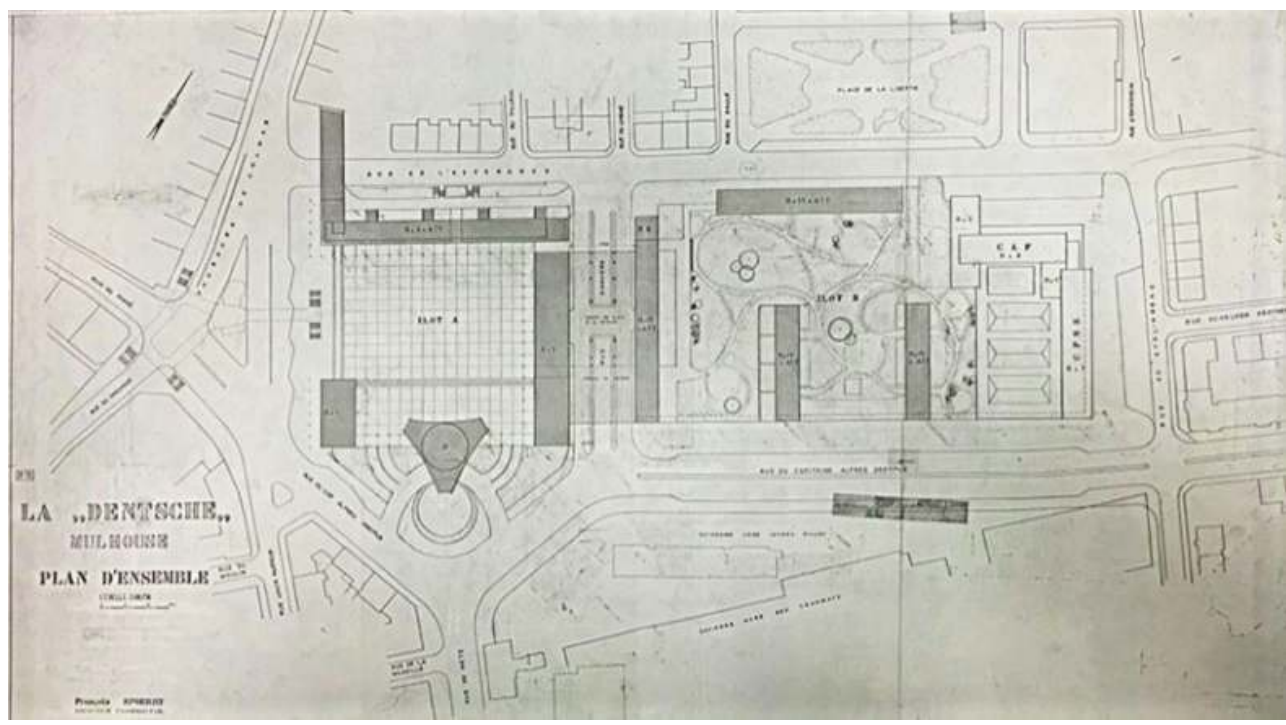

Plan-masse du projet de réaménagement du quartier de la Dentsche en quartier Europe, signé par François Spoerry, le 28 décembre 1962. Archives municipales de Mulhouse, fonds François Spoerry, Quartier de la Dentsche, 97C1001.

(C) Archives municipales de Mulhouse (AM M)

6 Le plan proposé par Spoerry et Calsat est accepté par le conseil municipal ${ }^{20}$ et n'est que peu modifié dans sa version finale, en 1962 (fig. 2). Celle-ci combine les idées préalablement esquissées par l'un ${ }^{21}$ et par l'autre ${ }^{22}$ : une composition unitaire articulant la place de forme carrée et une tour dessinées par Spoerry, quatre barres parallèles de logements au sein d'un jardin et une autre, placée perpendiculairement par Calsat, venant clôturer le jardin et minimiser le rapport entre la place de la Liberté et l'îlot. La liaison entre l'ancienne ville et ce nouveau fragment urbain prend corps à travers deux chevauchements de la rue de l'Espérance ${ }^{23}$ et un petit édifice créé sur la place de la Liberté (fig. 3). Spoerry, dans l'un des rares commentaires qu'il fait de ce projet, souligne l'importance de la place publique, qu'il imagine comme «le cœur de la ville ${ }^{24}$ » et qui, « entourée de vastes portiques [...] devrait constituer, pour les piétons, un lieu de détente leur donnant un sentiment d'espace qui leur manque dans le reste de la ville et où ils éprouveraient un sentiment de sécurité loin des voitures et du bruit de la circulation ${ }^{25}$ ». À l'issue de ce premier contrat, Spoerry est nommé responsable d'un organe de coordination mixte et équilibré créé en juin 1958; il est notamment chargé de "déterminer le style architectural de l'ensemble [et] d'harmoniser les éléments décoratif ${ }^{26} »$. Le mandater pour cette mission semble aller de soi en raison de son engagement - professionnel et politique auprès d'Émile Muller - et de sa connaissance du projet d'ensemble ; la réalisation de la tour, reconnue comme une « idée originale ${ }^{27}$ » de l'architecte, lui est ainsi confiée. 


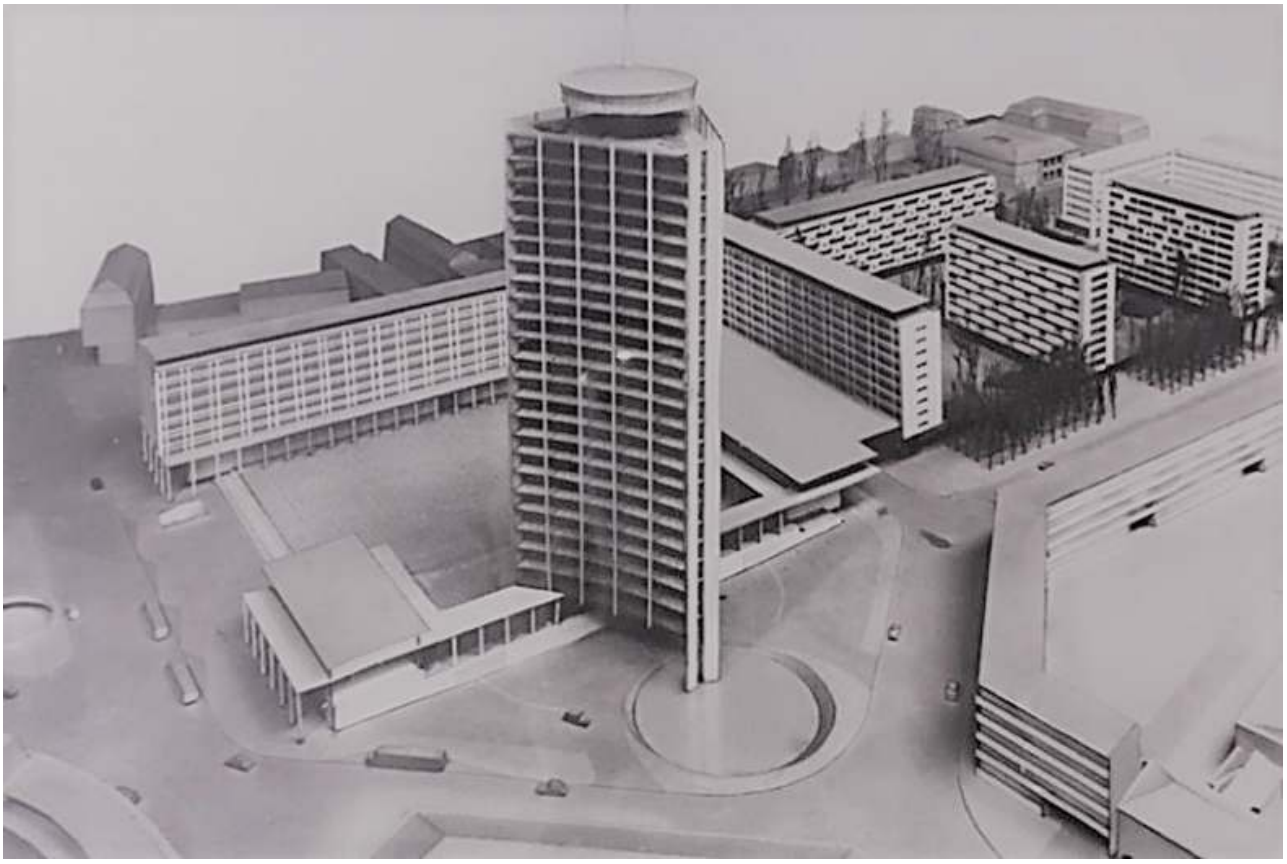

Maquette présentant la rénovation de l'îlot Dentsche, réalisée par Claude Harang, s.d., AM M, fonds S.A. Dentsche, $87 \mathrm{TT} 108,42 \mathrm{Fi} 2$.

(c) AM M.

7 La cohésion et les choix de la municipalité expliquent l'efficacité avec laquelle le projet d'aménagement du quartier Europe et de sa tour est élaboré28. Préparée et pilotée conjointement par la Ville de Mulhouse et la Société industrielle, la chambre de commerce et l'État, la « Journée mulhousienne de l'urbanisme » du 12 mai 1959 permet de médiatiser et valider les projets en gestation (fig. 4, fig. 5). La création du quartier Europe marque le coup d'envoi d'une série de projets destinés à faire entrer Mulhouse dans le $\mathrm{xx}^{\mathrm{e}}$ siècle, telle que la « ZUP » (zone à urbaniser en priorité) qui, lancée en 1964 lors du second mandat de Muller, doit accueillir sur 225 hectares de surface des ensembles sportifs, universitaires et résidentiels ${ }^{29}$. La présence du ministre de la Construction, Pierre Sudreau (1919-2012), participe au retentissement de cette journée qui se voit prolongée à travers le «Jour mondial de l'urbanisme", l'année suivante, le 8 novembre 1960. En obtenant que cet événement ait lieu à Mulhouse, le maire Muller consacre l'image d'une ville en pleine expansion au cœur de l'Europe qu'il a l'ambition de donner à la métropole haut-rhinoise. 
Figure 4

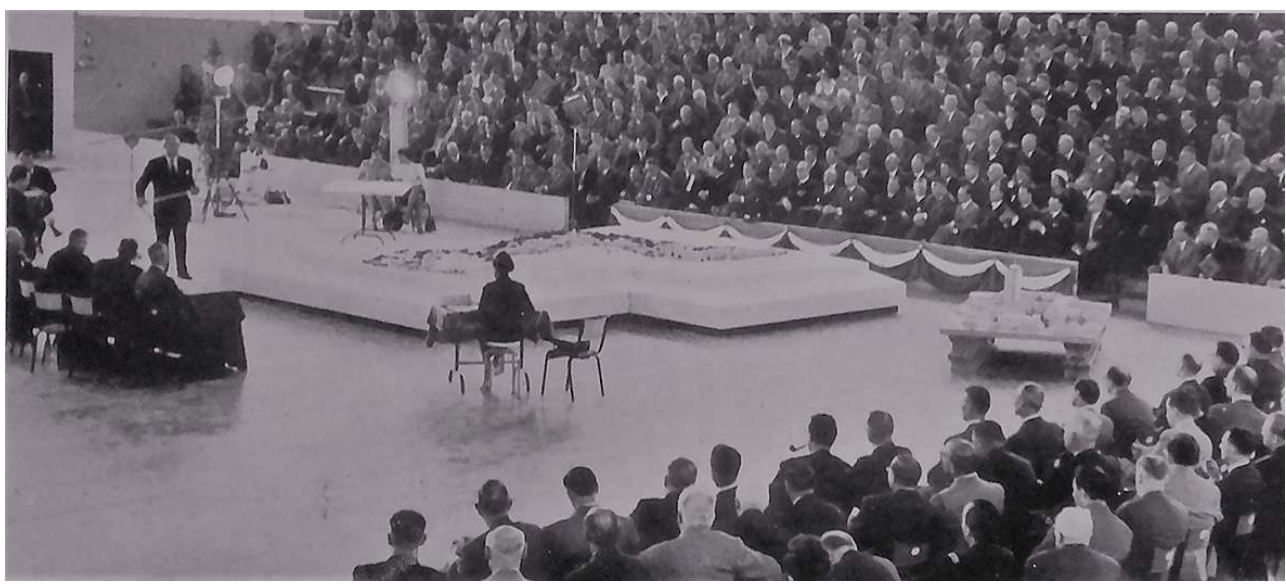

Présentation de la maquette du projet de réaménagement du site de la Dentsche à l'occasion de la Journée mulhousienne de l'urbanisme, le 12 mai 1959. Photographie depuis la salle des sports. Phot. anonyme (Bulletin de la société industrielle de Mulhouse, n 695, numéro spécial « Journée mulhousienne de l'urbanisme, mai 1959 », p. 21).

Figure 5

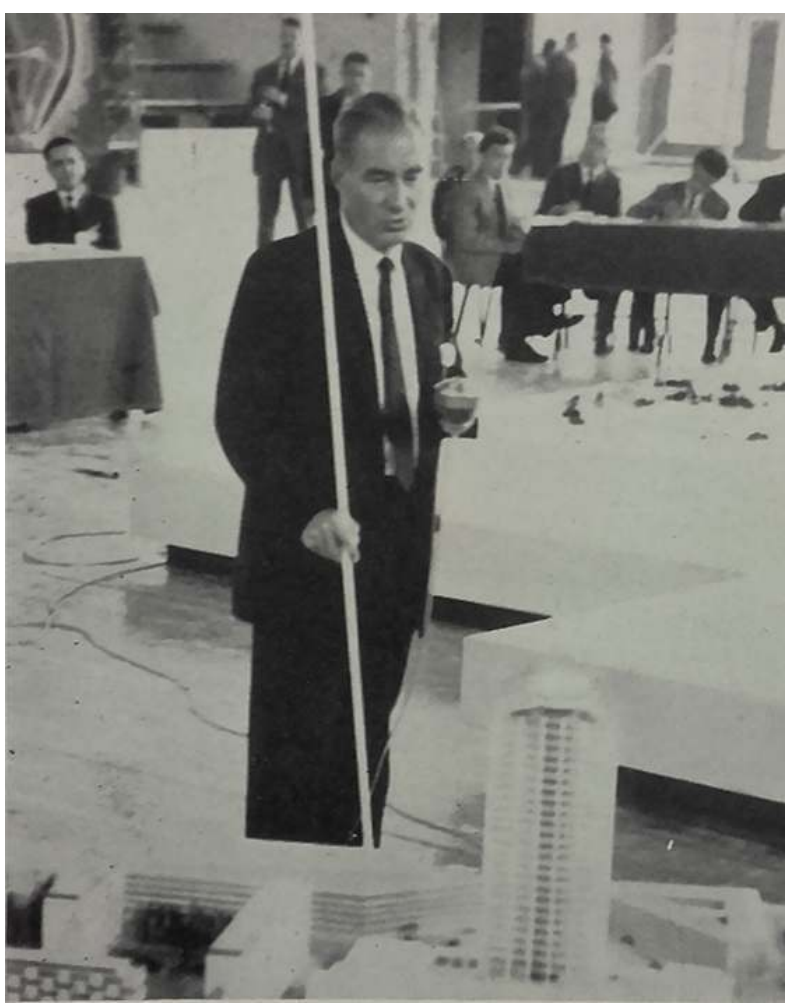

François Spoerry présentant la maquette du projet de réaménagement du site de la Dentsche à l'occasion de la Journée mulhousienne de l'urbanisme, le 12 mai 1959. Photographie depuis la salle des sports. Phot. anonyme (Bulletin de la société industrielle de Mulhouse, n 695 , numéro spécial « Journée mulhousienne de l'urbanisme, mai 1959 », p. 32). 


\section{Le symbole d'une tour européenne... de logements}

En 1957, la signature du traité de Rome confère à l'Europe une dimension nouvelle en redéfinissant ses contours politiques et économiques. Muller, engagé dans le débat politique européen - il est membre du Conseil de l'Europe (1958-1962) et membre du Parlement européen (1973-1979) - considère le projet de réaménagement de la Dentsche comme une vitrine et lui attribue, symboliquement, un aménagement artistique sur le thème de l'Europe. Sur la grande place minérale conçue pour devenir le lieu de rencontre des Mulhousiens, nommée "place de l'Europe", sont exposés des blasons de villes européennes ${ }^{30}$. Les rues bordant ce nouveau quartier prennent de nouvelles appellations ${ }^{31}$ et les bâtiments du quartier portent des noms en rapport avec l'Europe ${ }^{32}$. La tour de l'Europe (fig. 6), élément majeur de la composition urbaine, est porteuse de symboles : architecturalement, ses trois façades concaves reposant sur une base de plan triangulaire symbolisent l'union tri-nationale entre la France, l'Allemagne et la Suisse (fig. 7); politiquement, son inauguration a lieu le 5 mai 1973 , jour du $25^{\mathrm{e}}$ anniversaire de la création du Conseil de l'Europe.

Figure 6

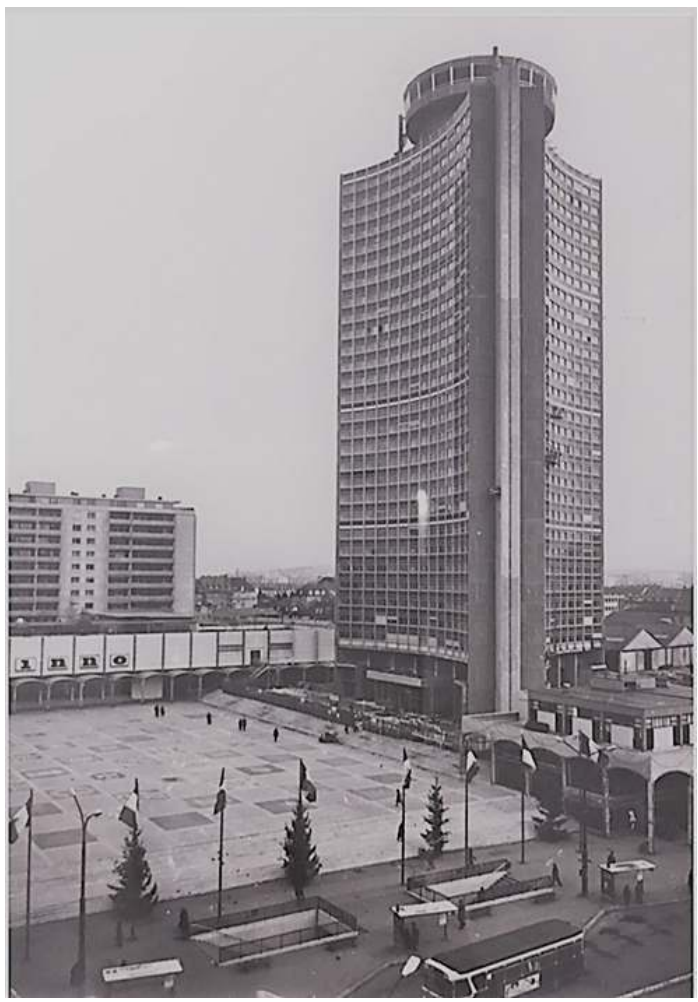

La tour de l'Europe, 1972. AM M., fonds S.A. Dentsche, 87TT108, 42Fi17.

Phot. anonyme. (C) AM M. 
Figure 7

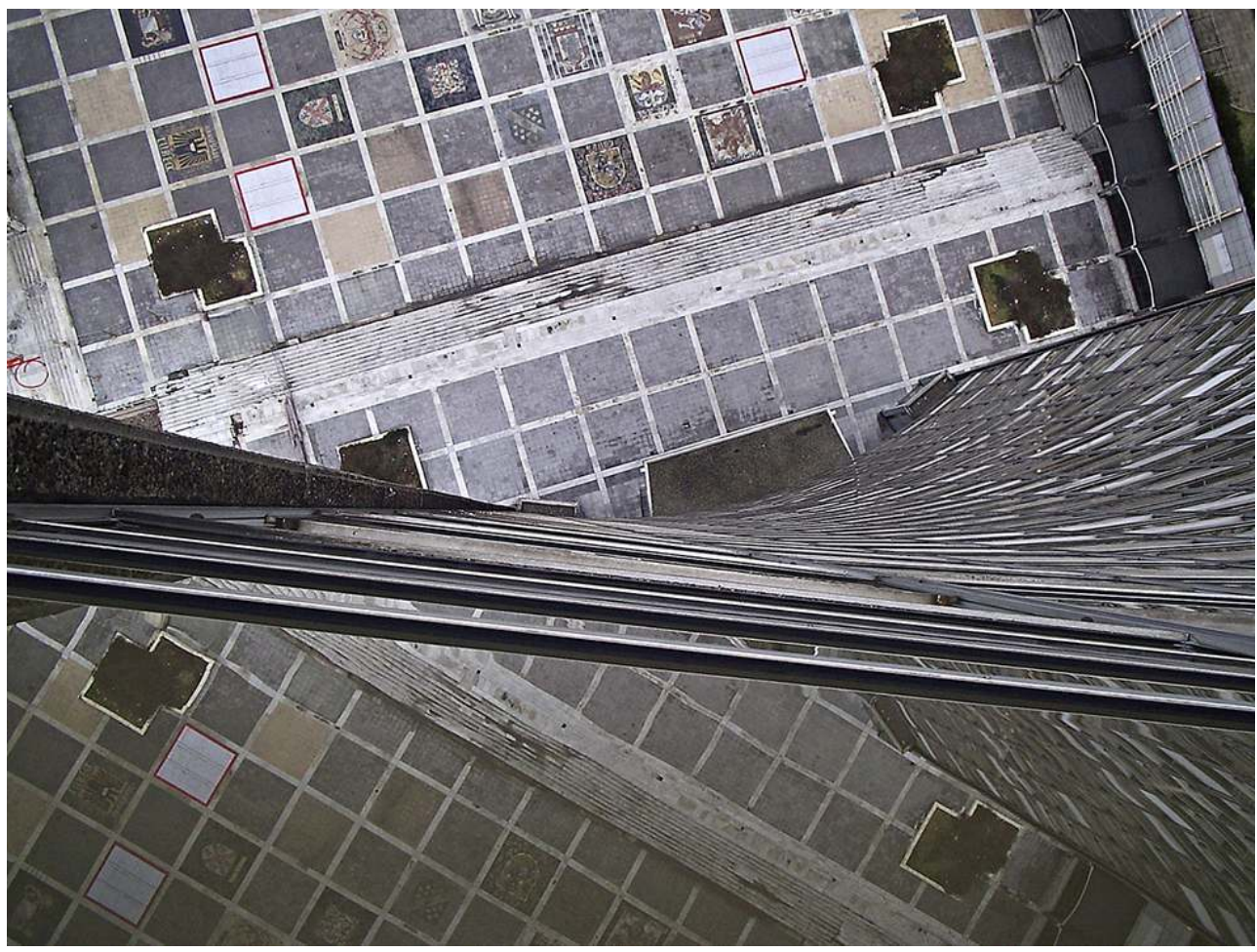

Vue plongeante sur une façade de la tour de l'Europe depuis un étage supérieur, 2006.

Phot. Géraldine Bouchet-Blancou. (c) Géraldine Bouchet-Blancou.

9 Lovée dans la mouvance d'un urbanisme vertical véhiculé dans les années 1960-1970 et répondant aux ambitions modernes, la tour de l'Europe de Mulhouse entre dans l'histoire de l'évolution de la tour européenne ${ }^{33}$. Inscrite dans le sillon de la tour Pirelli à Milan, figure pentagonale conçue par Luigi Nervi (1891-1979) qui incarne le renouveau économique de l'Italie, elle joue le rôle d'un «nouvel instrument urbain au profit d'une modernité sociale ${ }^{34}$ » et évoque des «symboles d'appartenance à un nouvel espace politique en construction ${ }^{35} \%$. Surplombant Mulhouse à 112 mètres de hauteur, elle demeure la tour la plus haute de l'époque, avant l'achèvement de la tour Montparnasse à Paris. Pour les Mulhousiens, elle incarne un signal comparable à celui de la capitale, puisqu'ils se laissent aller à rêver de « voir Mulhouse du haut de la tour de l'Europe, un peu comme on regarde Paris du haut de la tour Eiffel $^{36}{ }$ (fig. 8). Par son échelle et sa matérialité, la tour adopte le «caractère monumental ${ }^{37}$ " exigé par le programme. Au même titre que les tours conçues par Cornelis van Eesteren (1897-1988) aux Pays-Bas, ou Raymond Lopez (1904-1966) et Michel Holley (1924-) à Paris, la tour de l'Europe de Spoerry obéit à une "logique de composition globale ${ }^{38}$ ». Selon l'architecte, l'esthétique de la tour doit s'exprimer par une géométrie triangulaire en forme d'étoiles à trois branches afin de se distinguer d'une « mode ${ }^{39}$ " des tours parallélépipèdes rectangles. En effet, si Spoerry confère à la tour une architecture résolument moderne - par sa technique et son langage - il désire toutefois s'éloigner de la «tyrannie intellectuelle des tenants de l'architecture moderne et, afin de pallier leur aveuglement, [...] réfléchir à une nouvelle renaissance ${ }^{40} »$. 
Figure 8

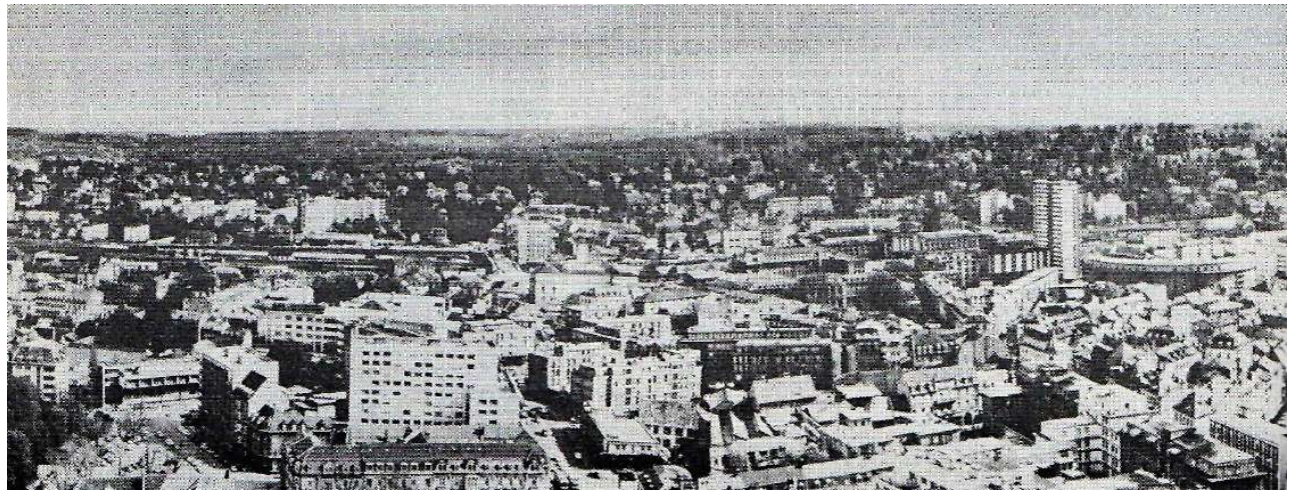

Vue panoramique vers le sud de Mulhouse depuis le dernier étage de la tour de l'Europe, s.d. (vers 1970). Phot. anonyme (JAQUEL, Roger, TROER, Charles, MEYER, Paul. Mulhouse plein cadre. Aspects d'une ville vue de la tour de l'Europe. Mulhouse : Omniatec, 1973, p. 6).

Initialement dédiée à l'activité tertiaire et planifiée pour accueillir des bureaux, la tour de l'Europe se transforme peu à peu en une tour à programme mixte de 191 lots dont 146 logements (environ $15000 \mathrm{~m}^{2}$ ), 45 lots de bureaux et commerces (environ $38000 \mathrm{~m}^{2}$ ). Modulable, le plan permet aux espaces d'accueillir tantôt les fonctions de bureaux, tantôt celles d'appartements (fig. 9).

Figure 9

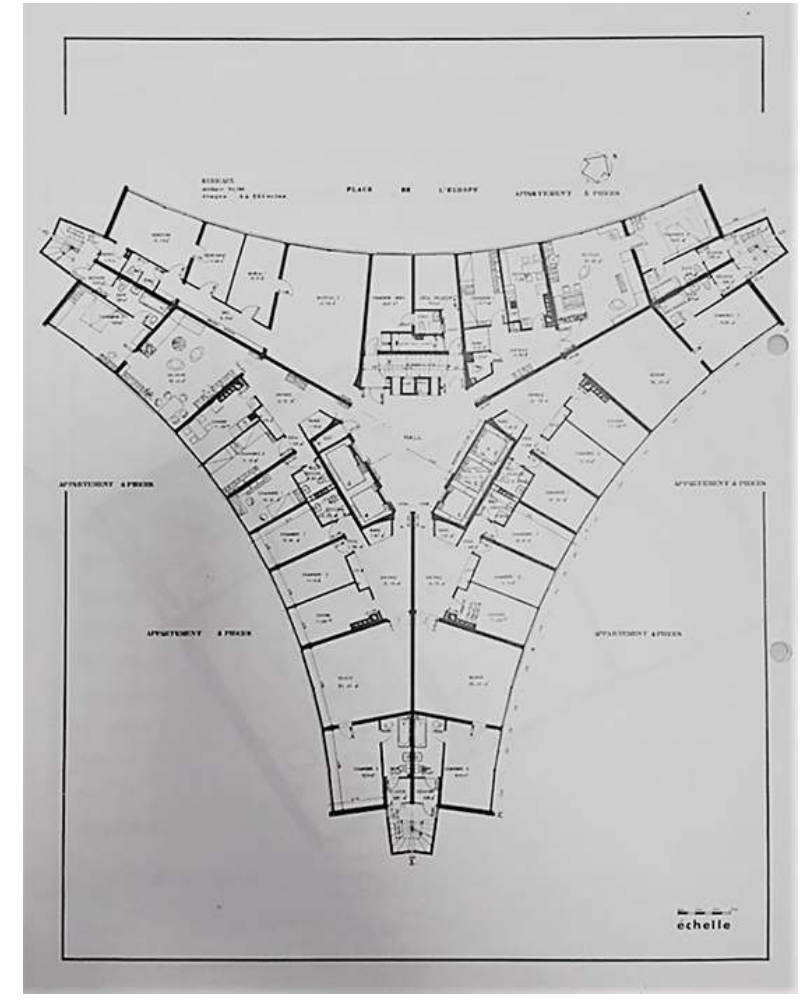

Plan de la résidence La tour de l'Europe, s.d., AM Mulhouse, fonds Bernard Michau, Fi14. (c) AM M.

11 Les six logements par niveau - répartis en deux logements de $106 \mathrm{~m}^{2}$ mono-orientés par branche - sont distribués par un noyau central de circulation lui-même secondé par un 
système d'escaliers de secours à l'extrémité de chaque branche. Avec son plan en Y, dont découlent la répartition des logements et leur orientation, la tour de l'Europe contribue aux recherches en matière de logements au même titre que le Aalto-Hochhaus de Alvar Aalto (1898-1976) à Brême, le Millefiori de Jean Ginsberg (1905-1983) à Monte-Carlo ou encore à la Gradelle de Hentsch \& Associates à Genève, respectivement achevés en 1962, 1963 et 1966. Du haut de ses trente étages couronnés par un restaurant à la vue panoramique, la tour de l'Europe entend ainsi incarner la modernité et l'identité européenne par le biais de l'habitat et non, comme c'est le cas dans la capitale alsacienne durant les mêmes années, par des édifices institutionnels directement voués à la question européenne. Figure héritière du « socialisme municipal ${ }^{41}$ » de l'entre-deux-guerres, Émile Muller place au cœur de son action la question du logement. La tour de l'Europe, si elle en est l'emblème le plus visible, s'inscrit dans un plan plus vaste. En 1964, le maire affiche un bilan positif de la politique en faveur du logement qu'il mène depuis dix ans: « 9000 logements neufs ont été édifiés, [...] et 1500 autres sont en chantier ${ }^{42}$ ». Les architectes Marcel Lods (1891-1978) et Maurice Novarina (1907-2002), figures de la Reconstruction et des grands ensembles en France, conçoivent en effet le plan d'urbanisme de la ZUP des Coteaux, sur les hauteurs de l'ouest mulhousien. Ce chantier pharaonique de plusieurs barres et tours de logements atteignant parfois 18 étages permet à la ville de Mulhouse d'accroître considérablement sa population, qui passe de 87000 habitants en 1946 à plus de 110000 en 1964.

\section{Un patrimoine transfrontalier en péril ?}

12 Dans l'euphorie des Trente Glorieuses, quand l'essor d'une société se jauge au nombre de mètres cubes de béton coulét ${ }^{43}$, Mulhouse se projette dans le futur. En 1961, la revue Saisons d'Alsace consacre un numéro spécial à une vision prospective de ce que sera la région dans dix ans. Le quartier de la Dentsche - qui en fait la couverture (fig. 10) - est en cours de transformation avec sa tour, ses bureaux d'affaires, ses arcades, ses magasins et parkings souterrains, et est rêvé comme "le cœur de la cité nouvelle ${ }^{44}$ ». Pauvre en monuments historiques, la ville affirme miser sur ces «audacieuses réalisations d'urbanisme ${ }^{45}$ » pour attirer les touristes, au détriment du centre-ville ancien qui dépérit ${ }^{46}$. Mais le nouveau centre, que l'on a voulu résolument moderne, ne répond pas aux exigences projetées. Les préoccupations fonctionnelles qui guident le travail des architectes - amélioration de la circulation, passages souterrains pour les piétons et stationnement enterré pour les voitures, jardins suspendus - répondent aux préoccupations dictées par la Charte d'Athènes ${ }^{47}$, manifeste du mouvement moderne publié quinze ans auparavant. Pourtant, ces solutions, si elles paraissent idéales sur le plan, déçoivent. L'urbanisme de dalle, implanté deux étages au-dessus du niveau de la rue passante, empêche tout lien avec son environnement ${ }^{48}$. Plus tard, l'adjonction d'éléments divers en bordure du site (gare centrale des transports en commun et d'un bureau de police, escalier au pied de la tour) referme peu à peu l'ensemble de la composition. Ces équipements concentrent la vie et participent au développement de la cité mais ils masquent aussi le parvis d'une place qui se voulait ouverte au monde, comme si «l'on n'osait plus attester de cet urbanisme ${ }^{49}$ ». 
Figure 10

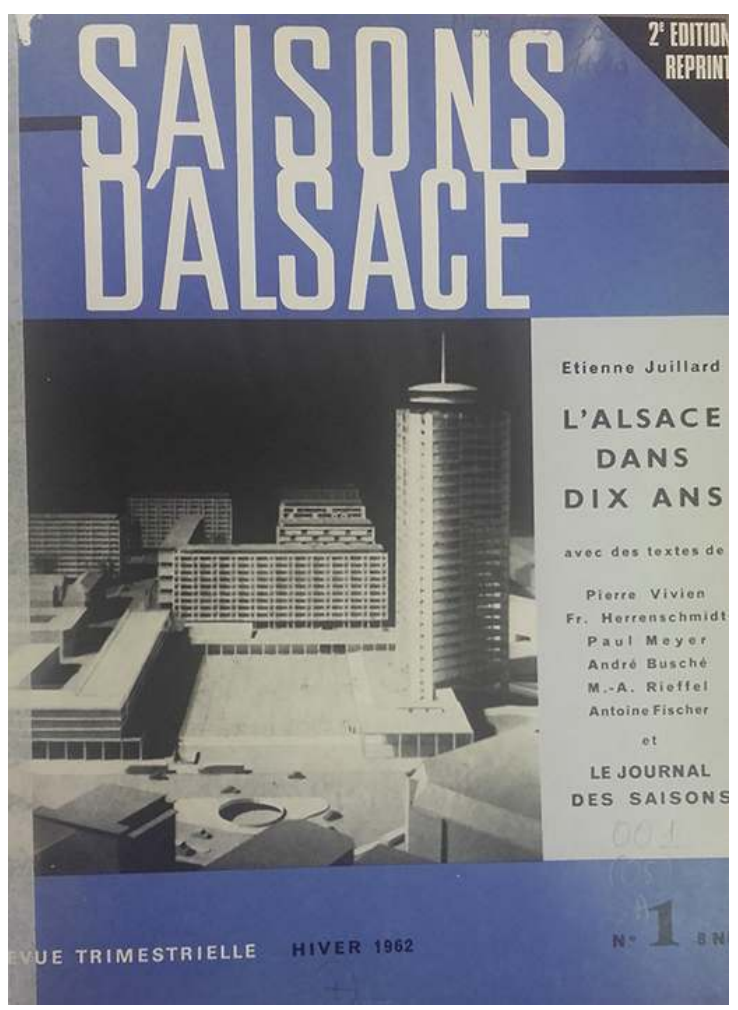

Page de couverture de la réédition du numéro spécial «L'Alsace dans dix ans » de Saisons d'Alsace de 1961 (Saisons d'Alsace, La Nuée bleue/DNA, 1991, réédition originale du nº 1 de janvier 1962).

La tour, objet qui incarne d'abord la marche du progrès et frappe l'imagination des architectes, connaît ensuite de vives critiques lors du choc pétrolier de 1973. La tour de l'Europe, si elle est le témoin des ambitions des années 1960, subit de plein fouet cette inversion de tendances dès son achèvement en 1972. La réception mitigée de l'opération auprès de la population locale trouve un écho dans l'attitude même de ses concepteurs. L'engagement de Spoerry semble en effet davantage dû au soutien qu'il témoigne à Émile Muller qu'à une volonté de produire un geste architectural puissant. Peu bavard à propos de la tour de l'Europe ${ }^{50}$, Spoerry avoue commencer « vraiment à mettre en pratiques [ses] idées architecturales ${ }^{51} »$ dans les projets de cités lacustres qui ont fait sa renommée. S'insurgeant contre les visions prométhéennes du modernisme, il fait le choix d'un " urbanisme de la continuité ${ }^{52}$ » en rupture avec les "doctrines des avant-gardes ${ }^{53}$ ". Comme en réaction au projet mulhousien, dont il assume difficilement la paternité, il consacre alors sa carrière à créer, selon ses mots, une architecture « douce, rassurante, raisonnable ${ }^{54} \gg$.

Fruit de l'architecture des Trente Glorieuses, la conception technique de la tour de l'Europe reflète un état des connaissances d'une période durant laquelle les objectifs de performance énergétiques étaient différents de ceux qui ont cours aujourd'hui ${ }^{55}$. Les appartements, dotés d'un certain standing, engendrent de lourdes charges de copropriété ${ }^{56}$, et le classement IGH (immeuble grande hauteur) et ERP (établissement recevant du public) de la tour l'assujettit à d'importantes normes de sécurité (ascenseurs, locaux techniques, réfection des paliers...). Pour ces raisons, le prix de l'immobilier chute de manière importante autour de $2015^{57}$. Cette mauvaise santé économique se traduit par le 
départ des occupants, auquel s'ajoute celui des professionnels pour motif fiscal, depuis l'ouverture de la zone d'aménagement concerté, du Trident, en zone franche. Selon Simone Ancelet, présidente du conseil syndical de la tour, près de $17 \%$ de l'immeuble était inoccupé au 31 décembre 2014 (soit 18 logements et 16 locaux professionnels) ${ }^{58}$.

Le regard porté sur Mulhouse et son opération d'aménagement du quartier Europe évolue depuis ces dernières années. En 2015, deux éléments concomitants distinguent l'attention portée à ce patrimoine. Le label « Patrimoine $\mathrm{xx}^{\mathrm{e}}$ siècle » est décerné à la tour de l'Europe ${ }^{59}$ ainsi qu'à l'opération Porte de Bâle, mais pas à l'opération Porte Jeune - place de l'Europe qui fait en revanche l'objet d'un projet urbain de réhabilitation/rénovation. Selon les termes de l'arrêté du 29 septembre 2015 portant déclaration d'utilité publique du nouveau programme de réhabilitation du Centre Europe à Mulhouse, ce dernier fait suite au « constat de dégradation de ce centre commercial situé dans le centre-ville de Mulhouse $^{60}$ ». En raison de son «manque d'entretien [...], d'une forte vacance des cellules commerciales et d'une insécurité grandissante ${ }^{61}$ " la requalification du Centre Europe s'avère nécessaire. Achevée en 2008 par l'agence TOA architectes associés, le centre commercial de la Porte Jeune veut incarner un nouveau vecteur de rayonnement et d'attractivité du quartier et se donne pour objectif de réussir ce que l'architecture des années 1960 projetait sans réellement y parvenir, c'est-à-dire connecter «les lieux, les époques, les populations ${ }^{62} »$ (fig. 11). La tour de l'Europe, qui a suscité quelques projets de rénovation restés à l'état de papier ${ }^{63}$, devrait connaitre des travaux de rénovation lui permettant de retrouver son plus bel éclat.

Figure 11

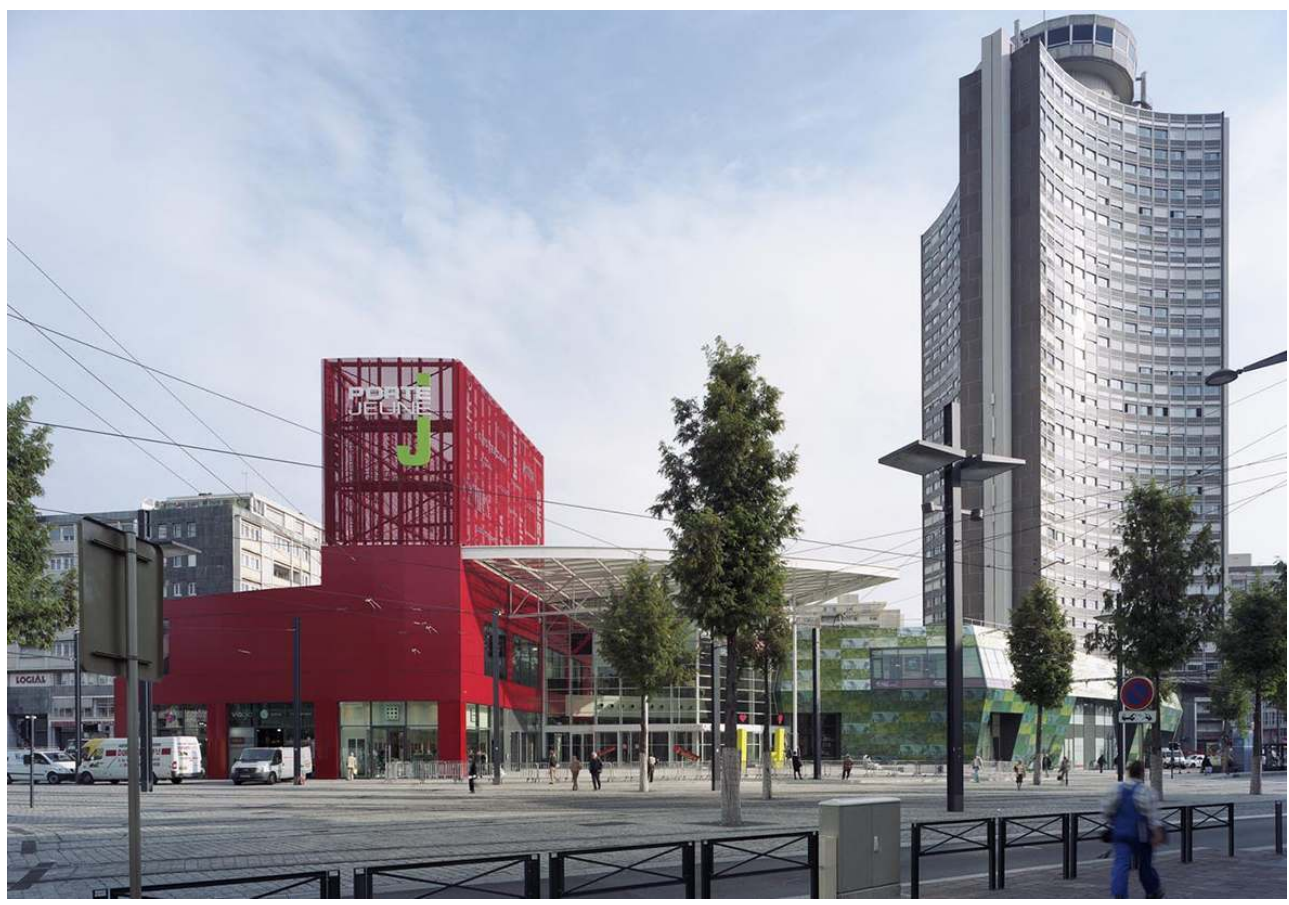

Image de synthèse du centre commercial Porte Jeune 68, s.d., TOA architectes associés. http://www.toa-archi.com/projets/tertiaire/centre-commercial-porte-jeune-68/

L'opération de reconversion du site de la Dentsche, lancée dans un contexte européen favorable, ouvre des perspectives nouvelles pour la ville. Avec son urbanisme de dalle et sa tour flottant comme le symbole d'une nouvelle cheminée dans le ciel mulhousien, 
l'opération peine à trouver une reconnaissance aux yeux du grand public et une place dans un système de valeurs culturelles largement partagées que le label « Patrimoine $\mathrm{xx}^{\mathrm{e}}$ siècle» entend alors conforter. Ce lent processus de patrimonialisation complique toutefois la lecture d'une opération urbaine plus large, voire «l'une des opérations d'urbanisme les plus abouties de l'après-guerre ${ }^{64} »$ : celle de la Porte de Bâle, du quartier gare et du quartier Europe dont la tour est l'élément saillant.

17 À la fin de la décennie 2000, la Ville de Mulhouse saisit l'occasion nouvelle d'occuper une place de choix dans le territoire transfrontalier en se portant candidate à l'Internationale BauAustellung (Exposition internationale d'architecture et d'urbanisme, IBA ${ }^{65}$ ). Développé en Allemagne au début $\mathrm{du} \mathrm{xx}^{\mathrm{e}}$ siècle pour promouvoir la culture allemande de planification, de construction et d'architecture, l'IBA nourrit désormais l'ambition de développer une culture de coopération transfrontalière en organisant des concours trinationaux visant, dans un temps défini, à inventer le futur d'un territoire par la réalisation de projets innovants et durables. Pour le concours «IBA Basel 2020 », dont le thème porte sur l'urbanisme et le paysage, Mulhouse entreprend la reconversion de 75 hectares du quartier DMC ${ }^{66}$, situés au cœur de la ville, en un quartier multifonctionnel. L'obtention du label «IBA Basel 2020 », reconnu internationalement, lui permettrait d'ouvrir une nouvelle page de son histoire.

\section{NOTES}

1. - Amandine Diener, architecte et docteure en histoire de l'architecture, est maître de conférences à l'Institut de Géoarchitecture de l'Université de Bretagne Occidentale et membre des laboratoires Géoarchitecture (EA 7462, UBO) et ARCHE (EA 3400, UDS). Elle prolonge ses recherches doctorales consacrées à l'histoire de l'enseignement de l'architecture à l'École des beaux-arts au xxe siècle, au sein du programme HEnsA20 (Histoire de l'Enseignement de l'Architecture au $\mathrm{xx}^{\mathrm{e}}$ siècle) dont elle assure la coordination et la valorisation. Elle contribue également aux recherches sur la production architecturale et urbaine des villes reconstruites et le développement de territoires métropolitains (POPSU Métropoles - Brest à la croisée des chemins).

2. - Émile Muller succède en 1956 à Jean Wagner, maire de Mulhouse élu en 1953 qui décède subitement. Il assure les responsabilités qui lui sont confiées jusqu'en 1959, date à laquelle il est élu maire.

3. - Compris entre les bornes chronologiques de 1740 et de 1848 , l'ouvrage de Stéphane Jonas épargne l'histoire de Mulhouse au-delà de son rattachement à la France; celui de Raymond Oberlé, bien qu'abordant l'histoire de la ville de manière complète, passe cependant rapidement sur la décennie de la fin des Trente Glorieuses; enfin, l'ouvrage d'André Heckendorn et JeanFrançois Mattauer retrace l'histoire de la ville à travers les actions des trois maires qui se sont succédé, Émile Muller, maire de 1956 à 1981, Joseph Klifa, maire de 1981 à 1989, et Jean-Marie Bockel, maire de 1989 à 2010. Voir JONAS, Stéphane. Le Mulhouse industriel. Un siècle d'histoire urbaine, 1740-1848. Paris : L'Harmattan, 1995 ; OBERLÉ, Raymond. Mulhouse ou La genèse d'une ville. Steinbrunn-le-Haut: Éd. du Rhin, 1985 ; HECKENDORN, André, MATTAUER, Jean-François. M ulhouse, une ville, trois maires. Mulhouse : Rugé, 2001. 
4. - Citons notamment BOEGLIN, Édouard. La Tête haute : 20 années, jour après jour. Mulhouse : Éd. du Caras, 1983 ; Id. Place de l'Europe, Genèse d'une ambition mulhousienne. Besançon : Cêtre, 2007.

5. - LAVAL, Henriette. Un nouveau centre urbain pour Mulhouse: De la Dentsche au quartier de l'Europe. Mémoire de master mené sous la direction d'Anne-Marie Châtelet. ENSA Strasbourg, 2016.

6. - Archives municipales, Mulhouse (AM M), fonds de la S.A. « la Dentsche », 1924-1996, 87TT.

7. - AM M, fonds François Spoerry, « Quartier de la Dentsche », 97C967 - 97C1462.

8. - AM M, fonds Bernard Michau, 1960-1980, 84TT.

9. - Notamment dans Saisons d'Alsace, «L'Alsace dans dix ans », hiver 1962, $\mathrm{n}^{\circ} 1$; « L'architecture en Alsace », été 1970, nº 35 ; « Où va l'Alsace », hiver 1990-1991, nº 110.

10. - Saisons d'Alsace, « Où va Mulhouse ?", automne 1997, $n^{\circ} 137$.

11. - Voir notamment BOLLE, Gauthier. «Reconstruire les paysages urbains et ruraux d'Alsace après 1945 : lignes de continuités ». Revue d'Alsace, 2016, nº 142, p. 117-138.

12. - HERRENSCHMIDT, François. "Flashes sur mille ans d'architecture en Alsace ». Saisons d'Alsace, été 1970, nº 35, p. 229-265, ici p. 262.

13. - Dès 1951, la Ville engageait le projet d'envergure de rénovation de son centre en procédant à l'acquisition d'un terrain de près de six hectares formant l'îlot Porte-Jeune/Dentsche, propriété des héritiers Schlumberger et de la Dentsche. Pour se donner les moyens de ses ambitions, la Ville crée également, en 1955, la Société d'économie mixte de rénovation urbaine de Mulhouse, chargée de l'aménagement du site. Actionnaire majoritaire, la Ville veille à la composition du conseil d'administration de cette société pour se garantir un pouvoir d'action et de contrôle sur le projet.

14. - François Spoerry fréquente l'école régionale d'Architecture en 1930 puis est admis à l'École des beaux-arts de Paris en 1937 après deux années de service militaire. Mobilisé entre 1939 et 1941, il poursuit ses études à l'école régionale d'Architecture de Marseille dans l'atelier d'Eugène Beaudouin et obtient son diplôme en 1943 pour le sujet «Un chantier naval ». Voir SPOERRY, François. L'Architecture douce, de Port-Grimaud à Port-Liberté. Paris : Robert Laffont, 1989, p. 128, et la notice de Spoerry dans le Dictionnaire des élèves architectes de l'École des beaux-arts (1800-1968) INHA, voir sur le site: http://agorha.inha.fr/inhaprod/jsp/reference.jsp? reference=INHA__METADONNEES__7 [consulté le 09/01/2019].

15. - SPOERRY, François. «L'itinéraire international d'un architecte mulhousien ». Les Annales de l'Académie d'Alsace, $3^{\mathrm{e}}$ trimestre $1984, \mathrm{n}^{\circ} 38$.

16. - « Rapport technique sur l'aménagement de la propriété dite la Dentsche à Mulhouse », s.d. (AM M, fonds S.A. « la Dentsche », 87TT101).

17. - Strasbourg, préfecture du département du Bas-Rhin et capitale de la région Alsace, se situe à une centaine de kilomètres de Mulhouse.

18. - «Contrat d'urbaniste-conseil pour l'établissement des plans-masses et d'aménagement du quartier de la Dentsche », $1^{\text {er }}$ février 1957 (AM M, fonds S.A. « la Dentsche », 87TT106).

19. - « Rapport technique sur l'aménagement de la propriété dite la Dentsche à Mulhouse », s.d. (AM M, fonds S.A. « la Dentsche », 87TT101).

20. - «Aménagement de la Dentsche : projet de base », 11 juin 1957 (AM M, fonds S.A. «la Dentsche », 87TT101).

21. - Deux propositions sont esquissées par Calsat en mars et juillet 1955. L'analyse de ces projets a été menée par LAVAL, Henriette. Op. cit., p. 21-22. Ces projets sont consultables dans le fonds de «la Dentsche », 87TT102, "Phase successive de réalisation du plan d'aménagement du quartier de la Dentsche, dessiné par les services d'urbanisme et d'étude de la ville de Mulhouse», mars 1955 ; et «Plan masse de l'aménagement du quartier de la Dentsche, dessiné à Paris le 12 juillet $1955 »$.

22. - Deux propositions sont esquissées par Spoerry ; si la première n'est pas datée (estimée à la fin de l'année 1955), la seconde date d'octobre 1956. L'analyse de ces projets a été menée par LAVAL, Henriette. Op.cit., p. 23-26. Ces projets sont consultables aux AM M, dans le fonds 
François Spoerry, Quartier de la Dentsche, «Ville de Mulhouse, étude du centre de la ville », s.d. [avant décembre 1956] (97C966), et « Plan d'urbanisme la Dentsche », 25 octobre 1956 (97C981). 23. - « Plan du quartier de la Dentsche au niveau du rez-de-chaussée », avril 1957 (AM M, fonds François Spoerry, Quartier de la Dentsche, 97C970).

24. - HECKENDORN, André, MATTAUER, Jean-François. Op. cit., p. 203.

25. - Ibid.

26. - Selon les termes du contrat, les missions confiées à Spoerry en tant qu'architectecoordinateur sont: "déterminer le style architectural de l'ensemble, [...] harmoniser les éléments décoratifs, [...] désigner les servitudes, [...] étudier les modes de fondations en fonction des sols, [...] assister, coordonner les architectes d'opération, [...] veiller aux prix des constructions, [...] coordonner les plannings afin de ne pas prendre de retard, [...] s'informer de la bonne réalisation finale des travaux». Voir AM M 87TT123, fonds S.A. «la Dentsche», Réaménagement du Quartier de la Dentsche par la société «la Dentsche », $2^{\mathrm{e}}$ partie - Mission de coordination, 14 juin 1958.

27. - Réaménagement du Quartier de la Dentsche par la société «la Dentsche », $2^{\mathrm{e}}$ partie Mission de coordination, 14 juin 1958 (AM M, fonds S.A. « la Dentsche », 87TT123).

28. - Plaquette « Mulhouse, 1953-1965. 12 années d'activités municipales », p. 3.

29. - « Mulhouse Demain ». Dernières Nouvelles d'Alsace, supplément octobre 1964.

30. - Les blasons des capitales européennes telles que Berlin, Paris, Rome, Luxembourg, Vienne et Bruxelles côtoient ceux de plus petites villes de province telles que Darmstadt, Düsseldorf, Fribourg-en-Brisgau, Karlsruhe, Mayence, Lille, Limoges, Nancy, Strasbourg et Nice.

31. - La rue du Capitaine-Dreyfus devient le boulevard de l'Europe et la rue de l'Espérance devient le boulevard Robert-Schuman.

32. - Citons la résidence Anvers, la résidence Robert-Schuman ou encore la tour de l'Europe.

33. - Voir TAILLANDIER, Ingrid, NAMIAS, Olivier, POUSSE, Jean-François. L'Invention de la tour européenne. Paris : Pavillon de l'Arsenal/Picard, 2009.

34. - Ibid., p. 33.

35. - ROTIVAL, Maurice. « Faut-il planifier l'Europe?». L'Architecture d'aujourd'hui, 1955, nº 63.

36. - JAQUEL, Roger, TROER, Charles, MEYER, Paul. Mulhouse plein cadre. Aspects d'une ville vue de la tour de l'Europe. Mulhouse : Omniatec, 1973, p. 3.

37. - « Rapport technique sur l'aménagement de la propriété dite la Dentsche à Mulhouse », s.d. (AM M, fonds S.A. « la Dentsche », 87TT101).

38. - NIVET, Soline. « Formes habitables ». Dans TAILLANDIER, Ingrid, NAMIAS, Olivier, POUSSE, Jean-François. Op. cit., p. 207.

39. - SPOERRY, François. L'architecture douce, de Port-Grimaud à Port-Liberté. Op. cit., p. 33.

40. - Ibid., p. 32.

41. - HECKENDORN, André, MATTAUER, Jean-François. Op. cit., p. 15.

42. - MULLER, Émile. « Mulhouse Demain ». Op. cit., p. 3.

43. - REUMAUX, Bernard. « Rendez-vous en l'an 2000 ». À propos de l'article introductif d'Étienne Juillard intitulé «L'Alsace dans dix ans », paru dans le $n^{\circ} 1$ de Saisons d'Alsace, hiver 1962, p. 4-41. Saisons d'Alsace, $\mathrm{n}^{\circ} 110$, hiver 1990-1991, rééd. originale du nº 1, p. 7.

44. - MEYER, Paul. "Mulhouse d'hier... et de demain ». Saisons d'Alsace, "L'Alsace dans dix ans ", hiver $1962, \mathrm{n}^{\circ} 1$, p. 77.

45. - Ibid.

46. - La reconquête du centre-ville, amorcée par Muller en procédant à certaines acquisitions, est approfondie par ses successeurs : Joseph Klifa (1931-2009) commence la piétonisation du centreville et met en chantier la cour des Maréchaux, finalement inaugurée par le maire Jean Marie Bockel, qui consacre le « renouveau du vrai cœur de ville » en piétonisant la place de la Réunion (voir HECKENDORN, André, MATTAUER, Jean-François. Op. cit., p. 203). 
47. - Citons notamment le point $\mathrm{n}^{\circ} 68$ de la Charte d'Athènes, qui souligne la possibilité, pour répondre aux exigences du développement de la cité, d'un « déplacement de centres considérés jusqu'ici comme immuables ». Voir LE CORBUSIER. La Charte d'Athènes [1941]. Paris : Éd. de Minuit, 1957.

48. - BRUANT, Benoît. "Méditations d'un promeneur ». Saisons d'Alsace, « Où va Mulhouse ?", automne 1997, nº 137, p. 141-147, ici p. 142.

49. - KLEINSCHMAGER, Richard. «Paysages urbains ». Saisons d'Alsace, «Où va Mulhouse? ", automne 1997, n 137, p. 153.

50. - Citons notamment les quelques lignes qu'il accorde à ce sujet dans son ouvrage «L'architecture douce » ainsi que dans son discours prononcé en 1984 lorsqu'il est invité par l'Académie d'Alsace à évoquer son parcours professionnel (voir SPOERRY, François. «L'itinéraire international d'un architecte mulhousien ». Art. cit.).

51. - SPOERRY, François. L'architecture douce, de Port-Grimaud à Port-Liberté. Op. cit., p. 35.

52. - Ibid., p. 124.

53. - Ibid.

54. - Termes employés par Spoerry dans son ouvrage L'Architecture douce, de Port-Grimaud à PortLiberté. Op. cit.

55. - Voir notamment SMART FRENCH. Le logement collectif du second $X X^{e}$ siècle, au prisme de l'énergie, programme interministériel de recherche et d'expérimentation, coordination : Raphaël Labrunye (GRIEF / ENSA-Bretagne), 2016-2018. Voir sur le site : http://aau.archi.fr/contrat-de-recherche/ smart-french-le-logement-collectif-du-second-xxe-siecle-au-prisme-de-lenergie/ [consulté le 10/01/2019].

56. - Selon Rémy Dantzer, directeur de Foncia Haut-Rhin et syndic de la tour de l'Europe à Mulhouse, la moyenne mensuelle des charges pour un appartement standard de $106 \mathrm{~m}^{2}$ est de 480 euros (voir MEICHLER, Frédéric. "Ma tour de l'Europe bien aimée ». L'Alsace, 16 octobre 2015).

57. - Selon Rémy Dantzer, le prix au mètre carré est descendu à 336 euros en 2015 (Ibid.).

58. - Ibid.

59. - Selon les critères définis par le ministère de la Culture, elle présente l'intérêt « architectural et urbain nécessaire [...] justifiant leur transmission aux générations futures comme des éléments à part entière $d u$ patrimoine $d u x^{e}$ siècle. Voir le site: http://www.culture.gouv.fr/ documentation/memoire/VISITES/labelxx/lieu_frameset.htm [consulté le 4 janvier 2018].

60. - Arrêté du 29 septembre 2015 portant déclaration d'utilité publique du nouveau programme de réhabilitation du Centre Europe à Mulhouse, Exposé des motifs et considérations justifiant le caractère d'utilité publique du projet du nouveau programme de réhabilitation de Centre Europe à Mulhouse, p. 3.

61. - Ibid.

62. - Voir le site: http://www.toa-archi.com/projets/tertiaire/centre-commercial-portejeune-68/ [consulté le 4 janvier 2018].

63. - En 2012, dans le cadre des Journées de l'architecture, l'association citoyenne " Mulhouse j'y crois " a permis à l'agence Arnaud Klein de présenter un projet de rénovation de la tour de l'Europe consistant en une redistribution des activités, un agrandissement des ouvertures de plain-pied, une extension des espaces extérieurs en terrasse, et un traitement lumineux renforçant le rôle de repère dans la ville sous une esthétique plus hi-tech. Voir sur le site : http:// arnaudklein.fr/24-tour-l-europe-2012.html [consulté le 4 janvier 2018].

64. - Propos recueillis lors d'une conférence de l'architecte Michel Spitz organisée le 6 octobre 2015 par l'université de Haute-Alsace, le Crédit mutuel et le journal L'Alsace, dans le cadre des Journées de l'architecture intitulée « Quel devenir pour la tour de l'Europe?». 
65. - « Internationale BauAustellung », littéralement " exposition d'architecture internationale », en français «Imaginer et bâtir l'avenir». Voir: http://iba-basel.net/fr/home [consulté le 07/02/2019].

66. - Du nom de l'ancienne entreprise textile Dollfus-Mieg et Compagnie, créée en 1746 par JeanHenri Dollfus.

\section{RÉSUMÉS}

Après un glorieux passé industriel, la ville de Mulhouse vit difficilement les mutations économiques des années 1930 et doit se reconstruire après la Seconde Guerre mondiale. Émile Muller (1915-1988), maire de la ville durant les Trente Glorieuses, entreprend d'audacieux projets urbains, parmi lesquels le projet de reconversion du quartier de la Dentsche en quartier «Europe ». Conçu selon les préceptes du mouvement moderne, cet urbanisme de dalle place au cœur de sa composition une tour conçue par l'architecte mulhousien François Spoerry (1912-1999) avec la collaboration de Bernard Michau (1928-). Inaugurée en mai 1973, elle symbolise la rencontre d'un territoire transfrontalier et alimente le débat sur le style en architecture. Contrairement aux édifices institutionnels qui voient le jour à Strasbourg durant les mêmes années, elle incarne l'expression d'une nouvelle identité culturelle et politique à l'échelle européenne par le biais du programme de l'habitat, et entre dans l'histoire de la tour européenne. À partir de fonds d'archives et de sources imprimées, cet article retrace la genèse de ce projet et mesure les ambitions qui ont motivé ses concepteurs, dans ses dimensions tant architecturales et urbaines que politiques. Il interroge également les limites d'un projet qui peine à trouver une reconnaissance aux yeux du grand public et une place dans un système de valeurs culturelles largement partagées, que le label « Patrimoine du $\mathrm{xx}^{\mathrm{e}}$ siècle », qui lui a été attribué en 2015, entend conforter.

After a glorious industrial past during the nineteenth century, the city of Mulhouse suffered from the economic changes of the 1930s and had to be rebuilt after the Second World War. Emile Muller (1915-1988), the city's mayor during the Trente Glorieuses, the post-war period of prosperity, undertook some audacious urban projects such as the conversion of the Dentsche neighbourhood into the Quartier Europe. Designed according to the tenets of the Modern Movement, this urban project, separating pedestrians from traffic by the creation of concrete platforms, had a residential tower block at the heart of its composition, designed by François Spoerry (1912-1999), a Mulhouse architect, working in association with Bernard Michau (1928-). Inaugurated in May 1973, this tower symbolised the coming together of a cross-border territory and generated much debate about architectural styles. Unlike the institutional buildings going up at Strasbourg during the same period, the tower, with its residential programme, embodied the expression of a new cultural and political identity in Europe. It also part of the history of tall buildings in Europe. From archival and published sources, this article looks at the genesis of this project and examines the geopolitical and architectural ambitions which inspired its designers. It looks too at the limitations of the project which, in the context of the general cultural values of the time, failed to win public appreciation. A label given to the twentieth-century heritage, «Patrimoine du xxe siècle» was conferred on the tower in 2015 and may help improve its appropriation by the local population. 
INDEX

Mots-clés : patrimoine, Trente Glorieuses, architecture, urbanisme, tour, modernité, frontière, Mulhouse, Europe, politique, style, label patrimoine

Keywords : heritage, 'Trente Glorieuses', architecture, town-planning, high-rise building, modernity, frontier, Mulhouse, Europe, politics, style, heritage label

\section{AUTEUR}

\section{AMANDINE DIENER}

Architecte et docteure en histoire de l'architecture, maître de conférences à l'Institut de Géoarchitecture de l'Université de Bretagne Occidentale, membre des laboratoires Géoarchitecture (EA 7462, UBO) et ARCHE (EA 3400, UDS) amandine.diener@gmail.com 